RUNNING HEAD: Aging and financial risk

\title{
Aging and financial exploitation risk
}

R. Nathan Spreng ${ }^{1,2,3,4}$, Natalie C. Ebner $5,6,7,8$, Bonnie E. Levin ${ }^{9}$, Gary R. Turner ${ }^{10}$

${ }^{1}$ Laboratory of Brain and Cognition, Montreal Neurological Institute, Department of Neurology and Neurosurgery, McGill University, Montreal, QC, Canada, nathan.spreng@gmail.com

${ }^{2}$ Departments of Psychiatry and Psychology, McGill University, Montreal, QC, Canada

${ }^{3}$ Douglas Mental Health University Institute, Verdun, QC, Canada

${ }^{4}$ McConnell Brain Imaging Centre, McGill University, Montreal, QC, Canada

${ }^{5}$ Department of Psychology, University of Florida, Gainesville, Florida, USA. natalie.ebner@ufl.edu

${ }^{6}$ Institute on Aging, Department of Aging \& Geriatric Research, University of Florida, Gainesville, Florida, USA.

${ }^{7}$ Cognitive Aging and Memory Clinical Translational Research Program, University of Florida, Gainesville, Florida, USA.

${ }^{8}$ Florida Institute for Cybersecurity Research, McKnight Brain Institute, \&

Pain Research and Intervention Center of Excellence, University of Florida, Gainesville, Florida, USA.

${ }^{9}$ Alexandria and Bernard Schoninger Professor of Neurology, University of Miami, Miller School of Medicine, Florida, USA. blevin@med.miami.edu

${ }^{10}$ Department of Psychology, York University, Toronto, ON, Canada, grturner@yorku.ca

Corresponding author:

R. Nathan Spreng

Montreal Neurological Institute

3801 University St.

Montreal, QC, H3A 2B4, Canada

p. 514-398-7268

e. nathan.spreng@gmail.com

To appear in:

Aging and Money, $2^{\text {nd }}$ edition

Ronan Factora (Ed)

Springer Press

Acknowledgements:

This work was supported by a pilot grant from the McKnight Brain Research Foundation and NIH grant 1R01AG057764-01A1. 


\begin{abstract}
Financial exploitation among older adults is an increasing public health concern. Whether in-person or online, financial risks are becoming omnipresent in our increasingly connected and wired world. Understanding why some older adults are more vulnerable to financial exploitation than others, and the specific contexts in which this vulnerability is most likely to emerge, is necessary to design more target surveillance and intervention tools. In this review, we identify the cognitive, socioemotional, and brain changes in aging that increase financial exploitation risks in later life. Next, we build on this review to propose a new social cognitive neuroscience model of financial exploitation risk. In the final section we review emerging work to develop profiles for older adults, towards novel intervention techniques to reduce the burden of financial exploitation in later life.
\end{abstract}

\title{
Keywords
}

Aging, financial exploitation, fraud, cyber security, cognitive aging neuroscience, socio-affective neuroscience, phishing, risk detection 


\section{Table of Contents}

I. Financial exploitation risk in older adulthood: Scope of the problem

II. Determinants of financial exploitation risk

Cognitive changes

Socioemotional changes

Structural and functional brain changes

III. Social cognitive neuroscience model of financial exploitation risk

IV. Financial risk: Assessment and surveillance

Structured Assessment of Financial Exploitation Risk (SAFER)

Online fraud and cyber-security risk assessments

V. Conclusions and future directions 
Among older adults financial loss due to reduced decision-making capacity and increased risk of deception has reached epidemic proportions, constituting an emerging public health crisis. Financial exploitation is now among the most common forms of elder mistreatment (1-5). A Senate report released in 2019 found older adults are losing an estimated \$2.9 billon annually to financial scams (6). Other estimates have reached as high as 36 billion (7). These numbers almost certainly underestimate the actual prevalence of fraud as many older adults are unaware or unwilling to report fraud for fear of appearing cognitively impaired or conforming to negative aging stereotypes (8). Further, the vast majority of exploitation cases involve family members or close acquaintances and are likely to go unreported or be ignored $(1,9)$.

These estimates, considered in the context of the rapid expansion of older adults living longer in the US and other industrialized nations based on projections reported by the Census Bureau, raise concern that financial decision-making deficits in older adulthood will impact an increasingly large segment of the population $(5,10)$. Going forward, financial exploitation of older adults is likely to impose increasing societal costs in terms of increasing health care and economic burdens (11). For older victims of exploitation, experiencing adverse financial events has been associated with greater rates of hospitalization and long-term care admissions, poor physical and mental health, and higher mortality (12, 13). Older adults acutely feel the effects of financial exploitation, with limited opportunity to recover from loss (14). Sudden and unexpected financial losses as a result of exploitation may force older adults onto public entitlement systems or relatives to take on unexpected financial burdens. Further, the realization of being a victim of fraud is often accompanied by feelings of self-blame, guilt and embarrassment. These stressors are widely recognized as adversely impacting psychological well-being. However, the specific toll of financial exploitation remains poorly studied and is likely underestimated (15).

At the same time, technological advances are opening up multiple avenues for poor financial decision-making, leading to scams and fraud in our increasingly wired world. In particular, phishing (e.g., via email, text, social media etc.) is a widespread threat and a leading tool for online fraud and subsequent financial exploitation. These attacks are particularly appealing to perpetrators because they are simple, readily available, low-cost to attackers and do not have to occur in mass-scale to be effective. The FBI received 2,400 complaints last year with victims' losses from phishing estimated at \$325 million (16). Surprisingly, older adults have largely been neglected in research on cyber-security and online fraud avoidance, even though they may be particularly at-risk for such attacks.

Whether in person or online, financial exploitation risk among older adults is emerging as a serious public health concern. These threats will surely increase in the coming decades as the baby boomer generation ages further into older adulthood, taking its place as the wealthiest generation in American history and providing an increasingly lucrative target population for financial fraud. Given the potential scope of the problem, identifying early indicators of financial exploitation risk is critically necessary (17). In the following sections we review the cognitive, socioemotional, and neural determinants of financial exploitation risk that are critically necessary to inform early surveillance efforts. We next present our social cognitive neuroscience model, identifying two pathways to financial exploitation risk in later life. These dual routes to exploitation risk recognize both cognitive and socioemotional factors, as well as the associated neural changes that occur in later life. We close the chapter with a review of financial risk assessment tools, and previewing our work to increase the efficacy of surveillance strategies. Together these efforts are focused towards the ultimate goal of early detection and intervention, to reduce the burden of financial exploitation in older adulthood. 


\section{Determinants of financial risk}

Demographic risk factors for financial exploitation include age, lower education and income levels $(18,19)$ as well as household size and race $(9,19,20)$. While these demographic factors are important for informing broader policy and public health initiatives, they do not address how to identify a given individual's risk for financial exploitation. Predicting person-specific risk profiles requires a better understanding of individual differences in cognitive and socioemotional capacities, as well as the social and motivational contexts within financial decisions are made in later life $(21,22)$. In a recent review, we proposed a novel model of decision-making in older adulthood incorporating cognitive, socioemotional, as well as the brain changes associated with the aging process (23). This model emerged from an earlier survey of the research literature, with particular consideration given to financial decisionmaking in everyday life, beyond the confines of the laboratory or the clinic. Real world financial decisions involve weighing concrete choices such as reconciling a weekly budget, managing investment risk profiles, or justifying a big-ticket purchase. Yet we often forget that these decisions are made in situ, in complex and shifting social milieu that require navigating emotional reactions and often nuanced social cues. Examples of these in situ dynamic social contexts could include the friendly local bank manager who is 'recommending' a higher risk (and higher fee) investment portfolio; the insurance telemarketer who calls repeatedly to promote 'no medical required' health insurance; or the grandson who continually asks to 'borrow' money. Enumerating the scope of financial risks confronting older adults in today's world, from calculating compound interest to calculating complex social agendas and motivations, requires broader consideration of both cognitive as well as socioemotional capacities. Understanding how these abilities evolve and manifest in the context of age related alterations in structural and brain function in later life will enable us to define increasingly sensitive risk profiles, improving detection, lessening risk, and ultimately help older adults to avoid fraud and exploitation.

Below we update our review of the recent scientific advances in mapping the cognitive, socioemotional and neural determinants of altered decision-making in older adults and suggest how these changes may relate to increased financial risk. Yet we urge caution here. It is important not to fall victim to the 'aging equals decline' heuristic. The portrayal of older adults as poor decision-makers, vulnerable to exploitation and abuse, continues to propagate through the popular media and in the scientific literature. However, this stereotype has surprisingly mixed empirical support. Older adults often show better or more adaptive decision-making abilities relative to their younger counterparts in many contexts, and these age-related gains often manifest in more real-world settings (10). Over the past decade the field of decision-science and, more recently, decision-neuroscience (24), has provided a more nuanced picture of the cognitive, affective, social, and neural determinants of decision-making capacity in older adults. Here we take care to highlight both the gains and the losses in cognitive and socioemotional functioning that occur throughout older adulthood, and how these contribute to differing - but not necessarily declining - financial exploitation risk profiles in later life.

Cognitive changes. Cognitive changes in older adulthood have been associated with financial exploitation risk. This has been demonstrated both in the context of age-related cognitive impairments $(25,26)$ as well as in normative aging $(3,22,27)$. Among the most robust patterns of age-related cognitive decline is a reduction in cognitive control, or 'fluid' cognitive abilities. These abilities are necessary to learn novel information, inhibit maladaptive responses, imagine and plan for the future, and to flexibly adapt one's behavior when the decision-making context changes $(28,29)$. In the context of financial exploitation risk, age-related declines in cognitive control have been associated with a more risky decision-making style, and declines in financial capacity - a critical risk factor for financial fraud and exploitation $(18,30)$. Poor cognitive control has been linked to risky decision-making in older adulthood. This risk profile in older adults has been directly associated with greater susceptibility to deceptive advertising, a key tool for fraudsters (31). Reduced cognitive control capacity has also been associated with poor decision-making, problem-solving and planning for one's financial future $(32,33)$. In addition 
to these more complex cognitive control abilities, declines in specific cognitive skills necessary for financial transactions have been associated with financial exploitation risk in aging and brain disease (32-34). Specific cognitive skills that have been investigated in the context of financial management include conceptual, pragmatic, and judgment abilities, ranging from basic (numericity) to more complex skills (financial reasoning and planning). These abilities are necessary to enumerate, allocate and monitor personal finances in the service of current and future financial goals (34).

However, not all cognitive processes decline with age. Knowledge of oneself and the world, often referred to as 'crystallized', conceptual, or semantic knowledge, continues to increase across the adult lifespan, remaining comparatively stable into older age (35). There is increasing evidence that older adults may be able to draw upon this expanded repertoire of semantic, or fact-based, knowledge to identify potentially fraudulent behavior or avoid exploitative situations $(10,24)$. In the context of financial decision-making, Li and colleagues (36) tested this possibility and reported that crystallized cognitive abilities in older adults directly offset losses in fluid abilities, leading to more adaptive financial decisions. Similarly, during a game in which players were required to make financial deals with their fellow game players, older adults rejected more 'unfair' offers than their younger counterparts (37). While younger adults incorporated more empathic responding into their deal-making strategies, older adults tapped into more lived experience (e.g., 'money doesn't buy friends') in making their decisions. While the older adults were as empathetic as the young, their reliance on personal experience and prior knowledge to make more 'rational' decisions took precedence.

These divergent trajectories of age-related cognitive changes, with fluid cognition decreasing and crystalized knowledge increasing, may also result in a mid-life 'sweet-spot' for optimal financial decision-making (38). While younger adults possess the cognitive control skills necessary to respond flexibly and adaptively to rapidly shifting financial contexts (including the motivations of others), they typically lack the experiential knowledge to identify longer-term patterns and anticipate future consequences. In contrast, older adults have lower fluid cognitive abilities, and reduced financial skills, leaving them vulnerable to exploitation. Yet, they are able to bring more lived experience and prior knowledge to decisions, allowing older adults to more accurately identify and potentially avoid longerterm risk. In the context of these shifting cognitive abilities across the adult lifespan, middle adulthood may provide the optimal balance of executive control and experiential knowledge to maximize financial decisions, in both the near and far term.

In sum, cognitive changes impact decision-making capacity across the lifespan. These changes have a direct impact on financial competence and magnify exploitation risk for older adults. Fluid cognitive abilities, necessary to make complex decisions in uncertain contexts decline. In contrast, greater reservoirs of prior knowledge in later life can be advantageous in contexts where past experience is a reliable predictor of future outcomes. Put simply, the decision-making context matters. Contexts involving speed, pressure, novel information, and shifting contingencies or agendas require high levels of cognitive control, and thus leave older adults at greater risk. In contrast, decision contexts that allow for deliberation, and the integration of current facts with prior knowledge, may be particularly advantageous for decision-making in aging.

Socioemotional changes. Fraud and financial exploitation, by definition, are transactional, i.e., they involve both victim and perpetrator. Avoiding fraudulent, exploitative or deceptive behaviors depends on the ability to successfully navigate complex, potentially conflict-ridden, social dynamics. In this context, reduced socioemotional capacity would be associated with heightened risk, both for interpersonal exploitation (the grandson who continuously 'borrows' money) and for more impersonal, albeit relational, forms of exploitation such as telemarketing fraud (39). Personal familiarity between victim and perpetrator almost certainly increases the social complexity of potentially exploitative situations. This may be a contributing factor for the overwhelming prevalence of within-family financial abuse $(1,9)$ and may be further compounded by diminishing social support and need for sociality, loneliness and a reduced sense of well-being $(26,40,41)$. 
There is growing evidence that socioemotional factors have different influences on decisionmaking in older versus younger adults. Older adults show reduced negative emotional arousal to anticipated losses (42). This absence of negative emotion to anticipated losses suggests that older adults may engage in more risky financial decision-making, and indeed this has been shown to be the case. Older adults make more sub-optimal decisions during risk-seeking (i.e. greater potential for loss), but not during risk-avoidant financial decisions (42). On a decision-making task designed to mimic real-world shifts in gain and loss contingencies, more than one-third of older adults did not identify altered contingencies and continued to make high-risk decisions (31). These older participants also failed to show anticipatory emotional arousal during risky choices. This suggests that a significant proportion of older adults may experience a disruption in deception-detection, although the evidence remains somewhat equivocal on this point.

Older adults also demonstrate less reactivity to negative emotional stimuli (see 43, for a review), including explicitly deceptive cues such as lies $(44,45)$ or breach of financial trust $(46)$. Reduced arousal to negative emotional cues has been associated with lower activation of a brain region involved in bodily sensation (47). This may mean that the 'gut-feeling' associated with a pending risky decision may be reduced with age (42). Altered emotional responsiveness to negative stimuli is also consistent with the well-documented 'positivity bias' in older adulthood. Older adults show selective attention to, and greater memory for, positively-valenced over negatively-valenced information (see 48, for a review). In the context of less detailed memories and representations of the future (49), it has been suggested that older adults may make decisions on the basis of a 'bright but blurry' future (50). Positively skewed, yet less precise, imaginings of the future, may heighten the risk of maladaptive long-term decisions.

Age-related emotional changes have direct impacts on decision-making abilities in social contexts, potentially increasing vulnerability to interpersonal exploitation (see 51, 52 for review). For example, older adults assign higher trustworthiness ratings to pictures of unknown faces than do young (47). They also show reduced flexibility in adjusting financial investment portfolios following a breach of trust by a partner in an investment game. In contrast, younger adults adapted their decision-making style and reduced investments following untrustworthy actions by game partners (46). Similar age differences in learning the trustworthiness of financial brokers has also been reported (53). Older adults display positivity biases in other social contexts, including impression formation. For example, they show particular difficulty overcoming positive first impressions such as matching trustworthy faces with untrustworthy behavior (53-56); leaving them vulnerable to the "wolves in sheep's clothing" (57). This may be pose a particular challenge for older adults encountering the friendly-looking, polite con artist who assumes a trustworthy appearance or reputation to mask untrustworthy behavior. It may also present an even greater challenge when assessing the motives of a known other, such as a cherished granddaughter, who's motives for 'borrowing' money may change from helpful to harmful over time.

Reduced ability to detect deception in aging has also been demonstrated in the cyber realm, in the context of an ecologically valid field experiment that simulated an email phishing campaign. Older women showed particular vulnerability to a cyber attack (58), and those very old individuals with low cognitive and affective functional profiles were particularly vulnerable (59). As noted earlier, studies investigating affective influences on decision-making suggest that older adults may adopt a more risky decision-making style in anticipation of a more positively imagined, albeit less detailed, future (50). These deficits also manifest in the social realm, broadening the specter of vulnerability from maladaptive personal choices to interpersonal exploitation and fraud. Further, age-related changes in socioemotional and cognitive processes likely interact to influence decision-making behavior. These interactions may be positive and beneficial in some contexts. As we described earlier, access to a larger repertoire of prior knowledge has been shown to mitigate impulsive decision-making and reduce temporal discounting during financial decision-making tasks (36). However, these changes may also compound deficits in more cognitively demanding contexts: older-adult decision-making is particularly impaired on tasks that require rule-learning or shifting contingencies, or involve more abstract or context-dependent reasoning 
(see 24), as well as the detection of negatively-valenced deceptive cues $(44,46,58)$, particularly when cognitive demands are high (60).

Structural and functional brain changes. In addition to the cognitive and socioemotional changes outlined above, researchers have examined whether changes in the structure and function of the brain predict financial exploitation risk in normal aging and brain disease. Such changes may provide early biomarkers, signaling increased financial exploitation risk in later life. Early work in this area (61) reported an association between reduced cortical volume in the angular gyrus of the parietal lobe in Mild Cognitive Impairment with performance on a comprehensive measure of financial skills and capacity the Financial Capacity Instrument (FCI 34). Performance on the FCI has also been associated with reduced dorsal medial prefrontal cortex volume in early Alzheimer's disease (62). Together these regions have been implicated in conceptual thinking as well as the ability to generate a mental image regarding one's future actions (63), two abilities that may be critical for fact-based financial planning, reasoning, and decision-making about monetary allocations.

Appraisal of socially-conveyed information in domains relevant to financial exploitation such as deceptive advertising $(31,64)$, trustworthiness judgments $(47,65)$, impression formation $(56,66)$, selfother judgments (67) or risky decision-making in novel contexts (68) has been consistently associated with medial prefrontal cortex structure and function. As reviewed briefly above, appraisal of information with high personal significance, or involving social inferences about the actions or intentions of others, is represented more dorsally in medial prefrontal regions, where the affective meaning of these contingencies is represented more ventrally (69). Differences in value expectation have been linked to alterations in an "Affective-Integrative-Motivational" brain circuit, including ventral striatum, anterior insula, medial temporal lobe and prefrontal cortex regions, as well as motor-planning areas (24). Medial prefrontal cortex is closely connected with limbic structures and ventral striatum, known to mediate affective responses and emotional regulation during decision-making tasks (24). The integrity of ventral frontal brain regions, and their connections to other brain systems, may be essential for navigating complex social dynamics in situations of high personal relevance, such as potential fraud or exploitation, particularly if the perpetrator is known (see also 52 for a recent extension of the "Affective-IntegrativeMotivational" framework to social decision making in aging).

Dorsal and ventral medial subregions of prefrontal cortex are core nodes in a network of brain regions collectively referred to as the default network (63). This assembly of functionally connected brain regions is engaged by internally-focused thoughts including thinking about one's past, an imagined future or mentalizing about the thoughts or feelings of others $(63,70,71)$. As noted earlier, the majority of financial exploitation incidents are transactional and occur in a social context $(1,9)$. Thus age-related brain changes to the default network may predict exploitation risk in older adulthood.

Our work has shown early evidence for this association (72). We recruited a group of older individuals who had been victims of financial exploitation and a closely matched sample of elderly adults

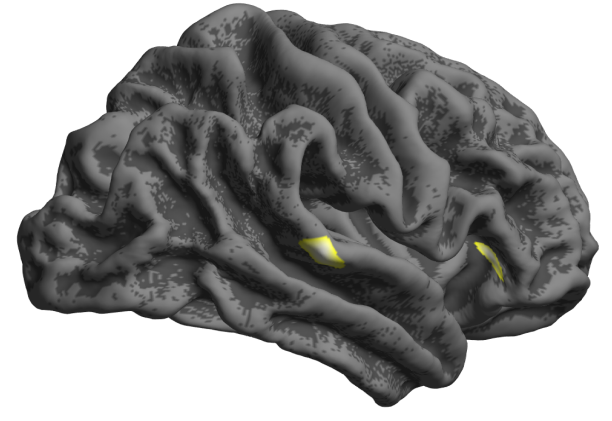

Figure 1. Reduced cortical thickness associated with financial fraud in the anterior insula and superior temporal sulcus/gyrus (right lateral hemisphere) who had been exposed to, but avoided, exploitation. All participants underwent structural and functional brain scans using magnetic resonance imaging (MRI) technology. Older adults who had experienced financial exploitation showed reduced anterior insula volume and increased functional connectivity between this region and the default network (see Figure 1). The anterior insula is a node in the salience network, a collection of brain regions implicated in detecting salient stimuli in the environment (73). This region has been implicated in generating our 'gut-feelings' in potentially deceptive contexts $(42,47)$ and is also part of the "AffectiveIntegrative-Motivational" brain circuit associated with decision-making capacity (24). Increased connectivity 
between salience and default network brain regions may signal greater reliance on more salient social processing during decision-making in older adulthood. Enhanced salience of social information, combined with altered social processing secondary to default network changes, such as positive impression formation (66), may leave older adults more vulnerable to undue social and affective influences and exploitation. Collectively, these findings suggest that changes in functional connectivity between large scale brain networks implicated in social decision-making may be an important neural marker of exploitation risk. We explore this idea further in our social cognitive neuroscience model described below (see Figure 2).

In this section we briefly reviewed the evidence linking age-related changes in cognitive, socioemotional, and neural function to decision-making ability and financial exploitation risk. Changes in each of these domains, and their interactions, directly influence exploitation risk in older adults. Accurately measuring these changes, and identifying clinically-relevant markers of exploitation risk, represents an important next step in early detection and intervention. In the next section, we present our social-cognitive neuroscience model of financial exploitation in older adulthood. This model is intended to provide a framework to guide future research on detection and intervention to reduce the risk of financial exploitation in later life.

\section{Social cognitive neuroscience model of financial exploitation}

Thus far we have highlighted the cognitive and socioemotional functions that increase vulnerability resulting in poor decision-making ability and ultimately exploitation risk. These include a shift from fluid intellectual abilities to greater reliance on experience and prior knowledge. Other changes include altered socioemotional functioning, including the emergence of a positivity bias, poor sensitivity to future financial loss, reduced deception detection, and poor trustworthiness judgments. Critically, these changes are associated with discrete patterns of functional and structural brain alterations in older adulthood, which in turn may signal context-specific vulnerabilities to financial fraud and exploitation in later life.

We have proposed an integrated social cognitive neuroscience model of financial risk in older adulthood. This model takes into account the complex changes described above as well as their associated neural mechanisms (Figure 2, 23). We reasoned that addressing the multi-dimensional factors underlying faulty decision making would yield a more comprehensive framework for developing surveillance and

assessment tools. We recognize the myriad determinants driving financial exploitation risk, some of which are fixed such as reading level, socioeconomic status, neighborhood factors, and ethnicity. They also include contextual factors such as financial regulatory regimes, availability of community and social as well as instrumental support, or access to educational and informational resources. However, our model focuses primarily on person-centered factors, such as neuropsychological and functional capacities, as mediated by age-related changes in brain structure and function. Based on the research reviewed above, we identify two behaviorally and neurally distinct pathways to increased financial risk in aging.

The first pathway originates from age-related changes in the balance between fluid intelligence abilities (which decline) and crystallized intelligence or prior knowledge (which is preserved). Loss of fluid cognitive abilities may lead to reduced efficiency in maintaining financial goals, inhibiting impulsive behaviors, or flexibly adapting to new contingencies or motivations, whether one's own or those of others. These changes can ultimately result in poor financial decision-making, consistent with past research $(18,41,74)$. The second pathway originates from reduced social capacity (specified in our model as social appraisal and perception) in older adulthood. As reviewed earlier, older adults show reduced ability to appraise and detect potentially deceptive information $(31,46,58)$. They also demonstrate alterations in social perception abilities necessary to make appropriate trustworthiness 
judgments $(55,56,64)$, and display increased attention to more positively-valenced information (48). These changes in socioemotional processing may leave older adults more vulnerable to undue social influences resulting ultimately in increased risk for exploitation at the hands of others, although research directly addressing this relationship remains in its infancy.

Critically, both pathways are associated with dissociable patterns of brain changes in older adults: Functional and structural changes in lateral frontal and parietal brain regions have been implicated in age-related decline in fluid reasoning ability such as working memory, inhibition and mental flexibility (75) and changes in socioemotional functioning are associated with alterations in the AffectiveIntegrative-Motivational brain circuit (24), reward circuitry (Suzuki et al., 2019), default network brain regions $(61,64,66,72)$, or their interaction. In developing this social cognitive neuroscience model of exploitation risk in aging, we put forth the idea that recognizing and appreciating these dissociable patterns of behavioral and brain change with age will help in the development of more context-specific

Cognitive capacity

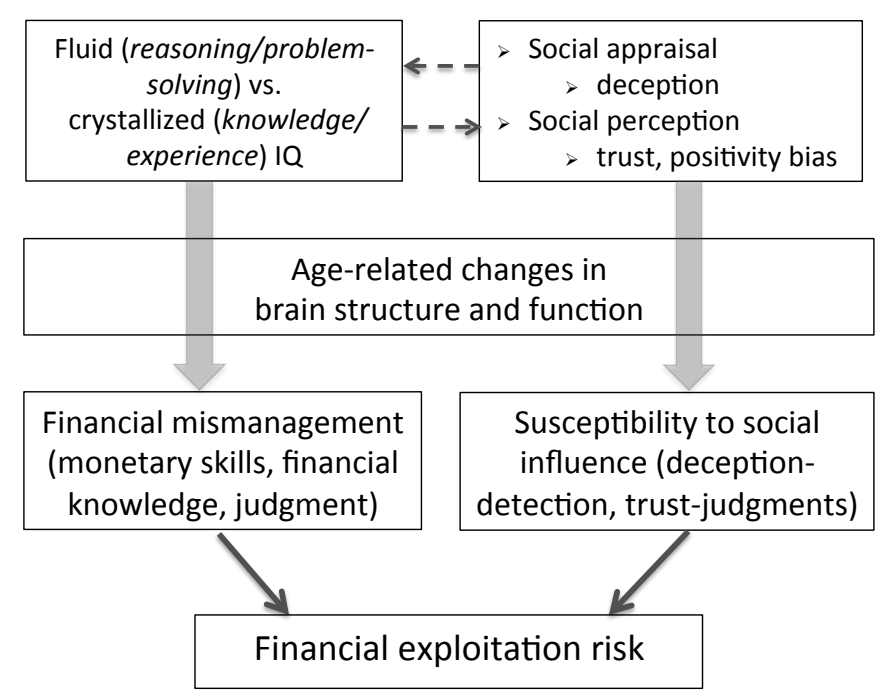

Figure 2. Social cognitive neuroscience model of financial exploitation risk indicators of financial exploitation risk in older adulthood (e.g., heightened vulnerability to exploitation in situations of high versus low social complexity).

As depicted in Figure 2, declines in cognitive capacity, associated with changes in lateral prefrontal cortex, would predict increased difficulty with financial management tasks such as problem-solving, planning or reallocation of resources. In contrast, declines in socioemotional capacities, associated with changes in default network brain regions, or their interactions with limbic regions and subcortical structures involved in affect processing, would lead to increased social vulnerability and exploitation at the hands of others. However the respective contributions of social and cognitive capacities to social decision-making remains an area of active study (60) and these factors almost certainly interact (represented by the reciprocal arrows in the top row of Figure 2). For example, previous research has shown that declines in working memory are associated with reduced capacity to mentalize about the thoughts or intentions of others (76). Similarly, working memory for social attributes of others is associated with interactions between default and frontal brain regions $(77,78)$. Thus, while there undoubtedly is coupling between social and cognitive capacities at the level of both brain and behavior, the intent of the model is to highlight and emphasize the distinct contributions of cognitive and socioemotional changes to financial risk and how these contribute to context-specific risk profiles for older adults.

Our review thus far has shown that patterns of cognitive and socioemotional functioning, as well as associated brain changes, in later life are well understood. However, adequate surveillance and intervention tools relating to these changes to financial risk profiles do not yet exist. In the next section we review existing surveillance tools as well as new directions in assessing financial risk emerging from our own laboratories. These efforts may lead to earlier identification of older adults at risk for financial exploitation, whether through financial mismanagement or undue social influence and coercion. 
An array of measures and assessment strategies have been developed to operationalize financial capacity and vulnerability to exploitation in older adults. These include self-report scales such as the Financial Industry Regulatory Authority Risk Meter (79); the Susceptibility to Scams Survey (18), and the Age Associated Financial Vulnerability Survey (40). Other self-report instruments measure basic skills such as financial literacy, numericity, and planning $(80,81)$. More recently, multi-dimensional assessments of financial capacity such as the Lichtenberg Financial Decision Making Rating Scale have been developed $(22,27)$. These assess both the contextual (previous history of exploitation, psychological well-being) and intellectual (decision-making capacity) factors that contribute to exploitation risk.

Self-report measures and assessment scales are the most widely available tools for assessing financial exploitation risk. However, comparatively few performance-based measures, necessary for more ecologically valid, in situ assessments, have been developed to measure financial skills and decision-making abilities. Among the most accessible and widely studied of these performance-based measures is the Financial Capacity Instrument (FCI, 34). The FCI was developed as a standardized measure to assess everyday financial skills. It is based on a multi-dimensional construct of financial capacity, encompassing discrete spheres of activity including basic monetary skills, financial conceptual knowledge, cash transactions, checkbook management, bank statement management, and financial judgment. The FCI has been used extensively to quantify declines in financial capacity, as well as providing an early behavioral marker signaling the onset and progression of brain disease $(32,34,82$, 83). In addition to measuring cognitively-dependent financial skills and abilities, our model suggests that performance-based assessments should also incorporate measures of socioemotional functioning. Moreover, these assessments should measure risk of in person exploitation as well as risk for the increasingly pervasive incidence of online fraud. Below we briefly outline our efforts to develop performance-based measures that are able to identify and distinguish cognitive and socioemotional determinants of financial risk, as well as the specific context in which that risk occurs, from personal decision-making to inter-individual exploitation, to online fraud.

Structured Assessment of Financial Exploitation Risk (SAFER), developed by Spreng and Turner, is a semi-structured interview based on the Assessment of Capacity for Everyday Decisionmaking (ACED). The ACED has been used to evaluate decision-making competence in cognitively impaired older adults $(84,85)$ We have designed a novel application of the instrument based on our model of financial exploitation risk (Figure 2). This new assessment tool is intended for use in a typically aging population to assess financial decision-making competence, and by extension, exploitation risk. A key feature of the SAFER is the ability to distinguish cognitive and social influences on financial decision-making. During the assessment, older adults are presented with two scenarios, drawn from real cases of financial exploitation. These scenarios were provided and vetted by a local adult protective services office. One scenario involves potential exploitation by an unknown other (credit card telemarketing) and a second involves a known other (theft by a family member). Decision-making competence is assessed along four dimensions: Understanding, problem appreciation, reasoning and decision-making logic. Older adults are asked a series of targeted questions to elucidate their reasoning and rationale for the decisions they make in response to each scenario. Separate scores are computed for decision-making capacity (quality of the response) and content (reliance on social versus non-social information). Early data are consistent with our model. Decision-making competence in these real-world contexts is determined both by the social context and the cognitive complexity of the decision.

The Assessment of Situational Judgment task (ASJ), developed by Getz and Levin (under review), examines one's ability to detect scams and infer deception. The ASJ is administered as an online survey and presents scenarios based on FBI cases as well as benign real-life requests for information. Categories of scams include invitations to participate in business opportunities, card skimmers, charity 
scams, emergency scams, game promotions/sweepstakes, government imposters and deceptive advertisements, identity theft, money transfer/check scams, and online/ phishing scams. In addition, subjects are asked to respond to questions pertaining to computer knowledge and scam exposure and experience. This tool was developed for older individuals and includes scams most used against the elderly.

Online fraud susceptibility and cyber security risk assessments. Training older adults to avoid online phishing attacks has limited efficacy, as skills are forgotten or poorly applied on-the-spot. This leaves older adults vulnerable to malicious phishing emails, even shortly after training (86). Based on our field-experimental work that suggested particular vulnerability to email-based phishing among older women (58) and older individuals with low cognitive and affective profiles (59), we identified an urgent need for behavior-based susceptibility profiles to inform intervention and fraud prevention strategies. Towards this goal we recently developed PEST -- the Phishing Email Suspicion Test -- an ecologically valid lab-based measure of phishing susceptibility (87). In PEST, participants rate a series of phishing and non-phishing emails regarding their level of suspiciousness. PEST allows quantification of email phishing susceptibility, as participants' overall level of suspicion regarding phishing emails and their ability to distinguish phishing from non-phishing emails. By comparing suspicion scores for each email to the email's real-world efficacy, we demonstrated the ecological validity of PEST. Phishing emails that were more effective in real-world behavior were also more effective at deceiving people in the laboratory. PEST is open-source and can be leveraged in private as well as corporate contexts to assist in profiling individuals according to their susceptibility risks. Using PEST we have recently demonstrated reduced ability to discriminate safe from malicious emails among cognitively unimpaired older adults (88). Further, this reduced discrimination ability more pronounced among older adults at increased genetic risk for cognitive decline secondary to Alzheimer's disease. Using PEST-based individual risk profiling, we are now able to build towards age-tailored/personalized defense solutions. These include decision-supportive monitoring and real-time warnings of potentially deceptive emails calibrated to individual risk profiles. Such personalized, automated, real-time cueing offers tremendous potential to assist in the decision-making process and reduce the burden of detecting deception for the individual, with the broader aim to reduce online financial fraud victimization.

Similar initiatives are emerging to provide older adults with information and guidance to reduce victimization in cyberspace. One recent initiative, supported by the Cybercrime Support Network (CSN) in partnership with Google, is ScamSpotter (https://scamspotter.org/). This platform was created to help particularly older adults to avoid getting swindled in the first place by focusing on "three golden rules" (Slow it down, Spot check, and Stop! Don't send), and was designed with seniors in mind to assure user friendliness (e.g., large font size, retro-inspired graphics, and ease of use for mobile devices and social media sharing).

\section{Conclusions and future directions}

As revealed in this brief review, age-related changes in decision-making capacity can directly influence financial competence and financial exploitation risk in older adulthood. Rapid advances in our understanding of the cognitive as well as the socioemotional affective, and social determinants of decision-making, and their neural basis, are opening new avenues for identifying person-specific predictors of exploitation risk. However, continued research is urgently needed to narrow the gap between investigations of neural mechanisms underlying decision-making deficits, and assessments of financial competence in the real-world, including cyberspace. The neural mechanisms associated with decision-making, and financial decision-making specifically, in older adulthood are becoming increasingly well-defined $(24,89)$. This is opening exciting opportunities for the development of more targeted, brain-based decision-making aids. The social cognitive neuroscience framework proposed here (Figure 2) is an important advance in this regard. Our model highlights dual paths to exploitation risk, 
differentiating cognitive and socioemotional changes, and their neural correlates. These two routes to risk may ultimately guide the development of more specific brain and behavioral markers, accounting for the specific contexts, in-person, online, in which exploitation is most likely to occur.

Another significant challenge in the field is to bridge the gap between laboratory measures and performance-based, real-world decision-making measures. Efforts are ongoing in this direction, including the development of ecologically valid assessment tools to measure financial competence (34, $84,85,90)$ and online fraud risks $(58,59,87,88)$ in vulnerable older adults. Consistent with our social cognitive neuroscience model, some of these instruments are also being incorporated into a neuroimaging environment to characterize the both the brain and behavioral correlates of performance on these tasks $(33,62)$.

While such advances are essential to mapping the neural and neurocognitive basis of financial exploitation risk, a critical missing link remains access to community-dwelling seniors who have been, or continue to be, financially exploited. This is a challenging yet urgently necessary research goal, to assess the discriminative validity of measures and markers of exploitation risk. To this end, greater collaboration between the research community and point of care service providers is warranted. Such collaborative efforts are needed to identify older adults who have experienced exploitation for participation in research projects, to vet and validate translational opportunities arising from the research, and ultimately to reinforce this first line-of-defense by providing community and financial services workers with the information and tools necessary to identify and prevent abuse before it occurs.

A White House Council on Aging report urged that much more research be conducted to improve surveillance and early detection of elder abuse (17). This will require large and diverse samples of older adults who have and have not been victims of exploitation to identify sensitive, specific, and generalizable behavioral and neural biomarkers of exploitation risk that will ultimately be translatable and useful for point of service providers. Large-scale, multi-site, and broadly-defined investigations are necessary. As we have shown here, much of this work is well underway. However efforts need to be rapidly scaled up to match the expanding scope of the problem of financial exploitation risk in our aging population. 


\section{REFERENCES}

1. Acierno R, et al. (2010) Prevalence and Correlates of Emotional, Physical, Sexual, and Financial Abuse and Potential Neglect in the United States: The National Elder Mistreatment Study. Am. J. Public Health 100(2):292-297.

2. Amstadter AB, et al. (2011) Prevalence and Correlates of Elder Mistreatment in South Carolina: The South Carolina Elder Mistreatment Study. J Interpers Violence 26(15):29472972.

3. Jackson SL \& Hafemeister TL (2011) Risk factors associated with elder abuse: the importance of differentiating by type of elder maltreatment. Violence Vict. 26(6):738-757.

4. $\quad$ FTC (2018) Federal Trade Commission, Consumer Sentinal Network Data Book, 2017 (Washington, D.C.).

5. Burnes D, et al. (2017) Prevalence of Financial Fraud and Scams Among Older Adults in the United States: A Systematic Review and Meta-Analysis. Am. J. Public Health 107(8):e13-e21.

6. $\quad$ AARP (2019) U.S. Senate Aging Committee Hearing Report on Financial Fraud (https://http://www.aarp.org/money/scams-fraud/info-2019/senate-aging-committeehearing.html).

7. True-Link (2015) The True Link Report on Elder Finanical Abuse. (True Link Financial, San Francisco, USA).

8. Pak K \& Shadel D (2011) AARP Foundation national fraud victim study. (AARP, Washington, D.C.).

9. Peterson JC, et al. (2014) Financial exploitation of older adults: a population-based prevalence study. J. Gen. Intern. Med. 29(12):1615-1623.

10. Ross M, Grossmann I, \& Schryer E (2014) Contrary to Psychological and Popular Opinion, There Is No Compelling Evidence That Older Adults Are Disproportionately Victimized by Consumer Fraud. Perspect Psychol Sci 9(4):427-442.

11. Weissberger GH, et al. (2020) Physical and mental health correlates of perceived financial exploitation in older adults: Preliminary findings from the Finance, Cognition, and Health in Elders Study (FINCHES). Aging \& mental health 24(5):740-746.

12. Button M, Lewis C, \& Tapley J (2014) No victimless crime: The impact of fraud on individual victms and their families. Security Journal 27(1):36-54.

13. Dong X \& Simon MA (2013) Elder abuse as a risk factor for hospitalization in older persons. JAMA internal medicine 173(10):911-917.

14. Nerenberg L (2000) Forgotten victims of elder financial crime and abuse: Facing the challenge. J. Elder Abuse Neglect 12(2):49-73.

15. Deliema M, Deevey M, Lussardi A, \& Mitchel OS (2017) Exploring the Risks and Consequences of Elder Fraud Victimization: Evidence from the Health and Retirement Study (December 1, 2017). Michigan Retirement Research Center Research Paper No. 2017-364, Wharton Pension Research Council Working Paper No. 2018-06, Available at SSRN: https://ssrn.com/abstract=3124952 or http://dx.doi.org/10.2139/ssrn.3124952.

16. FBI (2014) Internet Crime Complaint Center, Internet Crime Report (Federal Bureau of Investigation).

17. Pillemer K, Connolly MT, Breckman R, Spreng N, \& Lachs MS (2015) Elder mistreatment: priorities for consideration by the white house conference on aging. Gerontologist 55(2):320327.

18. James BD, Boyle PA, \& Bennett DA (2014) Correlates of susceptibility to scams in older adults without dementia. $J$ Elder Abuse Negl 26(2):107-122. 
19. Laumann EO, Leitsch SA, \& Waite LJ (2008) Elder mistreatment in the United States: prevalence estimates from a nationally representative study. J. Gerontol. B. Psychol. Sci. Soc. Sci. 63(4):S248-S254.

20. Beach SR, Schulz R, Castle NG, \& Rosen J (2010) Financial exploitation and psychological mistreatment among older adults: differences between African Americans and non-African Americans in a population-based survey. Gerontologist 50(6):744-757.

21. Lichtenberg PA, Ficker LJ, \& Rahman-Filipiak A (2016) Financial decision-making abilities and financial exploitation in older African Americans: Preliminary validity evidence for the Lichtenberg Financial Decision Rating Scale (LFDRS). J Elder Abuse Negl 28(1):14-33.

22. Lichtenberg PA, et al. (2018) Conceptual and Empirical Approaches to Financial Decisionmaking by Older Adults: Results from a Financial Decision-making Rating Scale. Clin. Gerontol. 41(1):42-65.

23. Spreng RN, Karlawish J, \& Marson D (2016) Cognitive, social, and neural determinants of diminished decision-making and financial exploitation-risk in aging and brain disease: A review and a new model. J. Elder Abuse Neglect 28(4-5):320-344.

24. Samanez-Larkin GR \& Knutson B (2015) Decision making in the ageing brain: changes in affective and motivational circuits. Nat. Rev. Neurosci. 16(5):278-289.

25. Boyle PA, Yu L, Schneider JA, Wilson RS, \& Bennett DA (2019) Scam Awareness Related to Incident Alzheimer Dementia and Mild Cognitive Impairment: A Prospective Cohort Study. Ann. Intern. Med. 170(10):702-709.

26. Lichtenberg PA (2016) Financial exploitation, financial capacity, and Alzheimer's disease. Am. Psychol. 71(4):312-320.

27. Lichtenberg PA, Gross E, \& Ficker LJ (2020) Quantifying Risk of Financial Incapacity and Financial Exploitation in Community-dwelling Older Adults: Utility of a Scoring System for the Lichtenberg Financial Decision-making Rating Scale. Clin. Gerontol. 43(3):266-280.

28. Park DC, Polk TA, Mikels JA, Taylor SF, \& Marshuetz C (2001) Cerebral aging: integration of brain and behavioral models of cognitive function. Dialogues in clinical neuroscience 3(3):151165.

29. Schacter DL, et al. (2012) The future of memory: remembering, imagining, and the brain. Neuron 76(4):677-694.

30. Boyle PA, et al. (2012) Poor decision making is a consequence of cognitive decline among older persons without Alzheimer's disease or mild cognitive impairment. PloS one 7(8):e43647.

31. Denburg NL, et al. (2007) The orbitofrontal cortex, real-world decision making, and normal aging. Ann. N. Y. Acad. Sci. 1121:480-498.

32. Griffith HR, et al. (2003) Impaired financial abilities in mild cognitive impairment: a direct assessment approach. Neurology 60(3):449-457.

33. Sherod MG, et al. (2009) Neurocognitive predictors of financial capacity across the dementia spectrum: Normal aging, mild cognitive impairment, and Alzheimer's disease. J. Int. Neuropsychol. Soc. 15(2):258-267.

34. Marson DC, et al. (2000) Assessing financial capacity in patients with Alzheimer disease: A conceptual model and prototype instrument. Arch. Neurol. 57(6):877-884.

35. Spreng RN \& Turner GR (2019) The Shifting Architecture of Cognition and Brain Function in Older Adulthood. Perspect Psychol Sci 14(4):523-542.

36. Li Y, Baldassi M, Johnson EJ, \& Weber EU (2013) Complementary cognitive capabilities, economic decision making, and aging. Psychol. Aging 28(3):595-613.

37. Beadle JN, et al. (2012) Effects of age-related differences in empathy on social economic decision-making. Int. Psychogeriatr. 24(5):822-833. 
38. Agarwal S, Driscoll JC, Gabaix X, \& Laibson D (2009) Age of reason: Finanical decisions over hte life cycle and implications for regulation. in Brookings Papers on Economic Activity - Fall, 2009 (Brookings Institute, Washington, D.C.), pp 51-117.

39. Pinsker DM \& McFarland K (2010) Exploitation in older adults: personal competence correlates of social vulnerability. Neuropsychology, development, and cognition. Section B, Aging, neuropsychology and cognition 17(6):673-708.

40. Lachs MS \& Han SD (2015) Age-Associated Financial Vulnerability: An Emerging Public Health Issue. Ann. Intern. Med. 163(11):877-878.

41. Shao J, Zhang Q, Ren Y, Li X, \& Lin T (2019) Why are older adults victims of fraud? Current knowledge and prospects regarding older adults' vulnerability to fraud. J Elder Abuse Negl 31(3):225-243.

42. Samanez-Larkin GR, et al. (2007) Anticipation of monetary gain but not loss in healthy older adults. Nat. Neurosci. 10(6):787-791.

43. Carstensen LL, et al. (2011) Emotional experience improves with age: evidence based on over 10 years of experience sampling. Psychol. Aging 26(1):21-33.

44. Ruffman T, Murray J, Halberstadt J, \& Vater T (2012) Age-related differences in deception. Psychol. Aging 27(3):543-549.

45. Stanley JT \& Blanchard-Fields F (2008) Challenges older adults face in detecting deceit: the role of emotion recognition. Psychol. Aging 23(1):24-32.

46. Frazier I, et al. (in press) Age and intranasal oxytocin effects on trust-related decisions: Behavioral and brain evidence. Psychol. Aging.

47. Castle E, et al. (2012) Neural and behavioral bases of age differences in perceptions of trust. Proc. Natl. Acad. Sci. U. S. A. 109(51):20848-20852.

48. Charles ST \& Carstensen LL (2010) Social and emotional aging. Annu. Rev. Psychol. 61:383409.

49. Addis DR, Wong AT, \& Schacter DL (2007) Remembering the past and imagining the future: common and distinct neural substrates during event construction and elaboration.

Neuropsychologia 45(7):1363-1377.

50. Weierich MR, et al. (2011) Older and wiser? An affective science perspective on age-related challenges in financial decision making. Social cognitive and affective neuroscience 6(2):195206.

51. Bailey PE \& Leon T (2019) A systematic review and meta-analysis of age-related differences in trust. Psychol. Aging 34(5):674-685.

52. Frazier I, Lighthall NR, Horta M, Perez E, \& Ebner NC (2019) CISDA: Changes in Integration for Social Decisions in Aging. Wiley interdisciplinary reviews. Cognitive science 10(3):e1490.

53. Rasmussen EC \& Gutchess A (2019) Can't Read my Broker Face: Learning About Trustworthiness With Age. J. Gerontol. B. Psychol. Sci. Soc. Sci. 74(1):82-86.

54. Bailey PE, Petridis K, McLennan SN, Ruffman T, \& Rendell PG (2019) Age-Related Preservation of Trust Following Minor Transgressions. J. Gerontol. B. Psychol. Sci. Soc. Sci. 74(1):74-81.

55. Suzuki A (2018) Persistent Reliance on Facial Appearance Among Older Adults When Judging Someone's Trustworthiness. J. Gerontol. B. Psychol. Sci. Soc. Sci. 73(4):573-583.

56. Suzuki A, et al. (2019) Age-related differences in the activation of the mentalizing- and rewardrelated brain regions during the learning of others' true trustworthiness. Neurobiol. Aging 73:18 .

57. Suzuki A \& Suga S (2010) Enhanced memory for the wolf in sheep's clothing: facial trustworthiness modulates face-trait associative memory. Cognition 117(2):224-229. 
58. Lin T, et al. (2019) Susceptibility to Spear-Phishing Emails: Effects of Internet User Demographics and Email Content. ACM transactions on computer-human interaction : a publication of the Association for Computing Machinery 26(5).

59. Ebner NC, et al. (2020) Uncovering Susceptibility Risk to Online Deception in Aging. $J$. Gerontol. B. Psychol. Sci. Soc. Sci. 75(3):522-533.

60. Zebrowitz LA, Boshyan J, Ward N, Gutchess A, \& Hadjikhani N (2017) The Older Adult Positivity Effect in Evaluations of Trustworthiness: Emotion Regulation or Cognitive Capacity? PloS one 12(1): 0169823.

61. Griffith HR, et al. (2010) Magnetic resonance imaging volume of the angular gyri predicts financial skill deficits in people with amnestic mild cognitive impairment. J. Am. Geriatr. Soc. 58(2):265-274.

62. Stoeckel LE, et al. (2013) MRI volume of the medial frontal cortex predicts financial capacity in patients with mild Alzheimer's disease. Brain imaging and behavior 7(3):282-292.

63. Andrews-Hanna JR, Smallwood J, \& Spreng RN (2014) The default network and self-generated thought: component processes, dynamic control, and clinical relevance. Ann. N. Y. Acad. Sci. 1316(1):29-52.

64. Asp E, et al. (2012) A neuropsychological test of belief and doubt: damage to ventromedial prefrontal cortex increases credulity for misleading advertising. Frontiers in neuroscience 6:100.

65. Zebrowitz LA, Ward N, Boshyan J, Gutchess A, \& Hadjikhani N (2018) Older adults' neural activation in the reward circuit is sensitive to face trustworthiness. Cogn Affect Behav Neurosci 18(1):21-34.

66. Cassidy BS, Leshikar ED, Shih JY, Aizenman A, \& Gutchess AH (2013) Valence-based age differences in medial prefrontal activity during impression formation. Social neuroscience 8(5):462-473.

67. Ochsner KN, et al. (2005) The neural correlates of direct and reflected self-knowledge. NeuroImage 28(4):797-814.

68. Samanez-Larkin \& Knutsen A (2014) Reward processing and risky decision making in the aging brain. The Neuroscience of Risky Decision Making, eds Reyna V \& Zayas V (American Psychological Association, Washington, D.C.).

69. Roy M, Shohamy D, \& Wager TD (2012) Ventromedial prefrontal-subcortical systems and the generation of affective meaning. Trends in cognitive sciences 16(3):147-156.

70. Spreng RN (2012) The fallacy of a "task-negative" network. Frontiers in psychology 3:145.

71. Spreng RN \& Mar RA (2012) I remember you: A role for memory in social cognition and the functional neuroanatomy of their interaction. Brain Res. 1428:43-50.

72. Spreng RN, et al. (2017) Financial Exploitation Is Associated With Structural and Functional Brain Differences in Healthy Older Adults. J. Gerontol. A. Biol. Sci. Med. Sci. 72(10):13651368.

73. Uddin LQ (2015) Salience processing and insular cortical function and dysfunction. Nat. Rev. Neurosci. 16(1):55-61.

74. Han SD, Boyle PA, James BD, Yu L, \& Bennett DA (2015) Mild Cognitive Impairment and Susceptibility to Scams in Old Age. Journal of Alzheimer's disease : JAD 49(3):845-851.

75. Turner GR \& Spreng RN (2012) Executive functions and neurocognitive aging: dissociable patterns of brain activity. Neurobiol. Aging 33(4):826 e821-813.

76. McKinnon MC \& Moscovitch M (2007) Domain-general contributions to social reasoning: theory of mind and deontic reasoning re-explored. Cognition 102(2):179-218.

77. Meyer ML \& Lieberman MD (2012) Social working memory: neurocognitive networks and directions for future research. Frontiers in psychology 3:571. 
78. Meyer ML, Spunt RP, Berkman ET, Taylor SE, \& Lieberman MD (2012) Evidence for social working memory from a parametric functional MRI study. Proc. Natl. Acad. Sci. U. S. A. 109(6):1883-1888.

79. FINRA (1999, 2020 online tool) Financial Industry Regulatory Authority Risk Meter (Financial Industry Regulatory Authority) https://tools.finra.org/risk_meter/.

80. Lusardi A \& Mitchell O (2007) Financial literacy and planning: Implication for retirement wellbeing. Business economics Jan:35-44.

81. Lusardi A \& Tufano P (2009) Debt literacy, financial experiences, and overindebtness. National Bureau of Economic Research. NBER Working Paper No. 14808.

82. Martin R, et al. (2008) Declining financial capacity in patients with mild Alzheimer disease: a one-year longitudinal study. Am. J. Geriatr. Psychiatry 16(3):209-219.

83. Niccolai LM, et al. (2017) Neurocognitive Predictors of Declining Financial Capacity in Persons with Mild Cognitive Impairment. Clin. Gerontol. 40(1):14-23.

84. Lai JM, et al. (2008) Everyday decision-making ability in older persons with cognitive impairment. Am. J. Geriatr. Psychiatry 16(8):693-696.

85. Lai JM \& Karlawish J (2007) Assessing the capacity to make everyday decisions: a guide for clinicians and an agenda for future research. Am. J. Geriatr. Psychiatry 15(2):101-111.

86. Caputo DD, Pfleeger SL, Freeman JD, \& Johnson ME (2014) Going spear phishing: Exploring embedded training and awareness. IEEE Security and Privacy 12:28-38.

87. Hakim ZM, et al. (in review) Evaluating the cognitive mechanisms of phishing detection with PEST, an ecologically valid lab-based measure of phishing susceptibility.

88. Grilli MD, et al. (in review) Is this phishing? Older age is associated with greater difficulty discriminating between safe and fraudulent emails.

89. Samanez-Larkin GR (2013) Financial Decision Making and the Aging Brain. APS observer 26(5):30-33.

90. Sawrie SM, Marson DC, Boothe AL, \& Harrell LE (1999) A method for assessing clinically relevant individual cognitive change in older adult populations. J. Gerontol. B. Psychol. Sci. Soc. Sci. 54(2):P116-124. 